\title{
Oculofaciocardiodental and Lenz microphthalmia syndromes result from distinct classes of mutations in $B C O R$
}

\author{
David $\mathrm{Ng}^{1}$, Nalin Thakker ${ }^{2}$, Connie M Corcoran ${ }^{3}$, Dian Donnai ${ }^{2}$, Rahat Perveen ${ }^{2}$, Adele Schneider ${ }^{4}$, \\ Donald W Hadley ${ }^{5}$, Cynthia Tifft ${ }^{6,7}$, Liqun Zhang ${ }^{2}$, Andrew O M Wilkie ${ }^{8}$, Jasper J van der Smagt ${ }^{9}$, \\ Robert J Gorlin ${ }^{10}$, Shawn M Burgess ${ }^{11}$, Vivian J Bardwell ${ }^{3}$, Graeme C M Black ${ }^{2,12,13}$ \& Leslie G Biesecker ${ }^{1}$
}

\begin{abstract}
Lenz microphthalmia is inherited in an X-linked recessive pattern and comprises microphthalmia, mental retardation, and skeletal and other anomalies. Two loci associated with this syndrome, MAA (microphthalmia with associated anomalies) and $M A A 2$, are situated respectively at Xq27-q28 (refs. 1,2) and Xp11.4-p21.2 (ref. 3). We identified a substitution, nt 254C $\rightarrow \mathrm{T}$; P85L, in BCOR (encoding BCL-6interacting corepressor, $\mathrm{BCOR}^{4}$ ) in affected males from the family with Lenz syndrome previously used to identify the $M A A 2$ locus $^{3}$. Oculofaciocardiodental syndrome (OFCD; OMIM 300166) is inherited in an X-linked dominant pattern with presumed male lethality and comprises microphthalmia, congenital cataracts, radiculomegaly, and cardiac and digital abnormalities. Given their phenotypic overlap, we proposed that OFCD and MAA2-associated Lenz microphthalmia were allelic, and we found different frameshift, deletion and nonsense mutations in $B C O R$ in seven families affected with OFCD. Like wild-type BCOR, BCOR P85L and an OFCDmutant form of BCOR can interact with BCL-6 and efficiently repress transcription. This indicates that these syndromes are likely to result from defects in alternative functions of BCOR, such as interactions with transcriptional partners other than BCL-6. We cloned the zebrafish (Danio rerio) ortholog of $B C O R$ and found that knock-down of this ortholog caused developmental perturbations of the eye, skeleton and central nervous system consistent with the human syndromes, confirming that BCOR is a key transcriptional regulator during early embryogenesis.
\end{abstract}

We had previously localized MAA2 to a $10-\mathrm{Mb}$ candidate region of $\mathrm{Xp}^{3}$. To identify the gene, we narrowed this region by excluding regions deleted in males with $\mathrm{Xp}$ deletions but without microphthalmia. Coriell cell line GM07947 is from a male with Duchenne muscular dystrophy, chronic granulomatous disease, McLeod phenotype and retinitis pigmentosa without microphthalmia ${ }^{5}$. Sequence-tagged site (STS) mapping confirmed previous data and oriented it to current genome maps. The deletion extended from $D M D$ (telomeric) to between RPGR and OTC (centromeric; Fig. 1). The Coriell cell line GM10283 excluded two genes telomeric to $D M D$. This narrowed the critical region to $\sim 5 \mathrm{Mb}$, including 12 identified genes not known to be mutated in humans. One of these, $D D X 3$, has an active $\mathrm{Y}$ homolog, and we therefore considered it unlikely to underlie a disorder with X-linked recessive inheritance. We sequenced the remaining 11 genes in members of the family in which MAA2 was originally identified. Ten of these genes showed no alterations, but we identified a missense change $(\mathrm{nt} 254 \mathrm{C} \rightarrow \mathrm{T}$ ) in $B C O R^{4}$ that co-segregated with the disease phenotype (lod score of 2.46, above the threshold of 2.0 for X linkage ${ }^{3}$ ). The corresponding residue of human BCOR, Pro85, is conserved in mouse, rat, chicken and pufferfish (Fig. 2), and we did not detect the nt $254 \mathrm{C} \rightarrow$ T mutation among $>450$ control chromosomes. X-inactivation studies of peripheral blood leukocytes in two carriers of the mutation showed no skewing (data not shown). We also sought, but did not find, $B C O R$ mutations in six males with sporadic microphthalmia. We did not find any mutations in BCOR in males from the two published families with MAA ${ }^{1,2}$ and linkage to MAA2 was excluded in both families (data not shown).

${ }^{1}$ Genetic Disease Research Branch, National Human Genome Research Institute, National Institutes of Health, Bethesda, Maryland, USA. ${ }^{2}$ Academic Unit of Medical Genetics and Regional Genetic Service, St Mary's Hospital, Manchester, UK. ${ }^{3}$ Department of Genetics, Cell Biology and Development and Cancer Center, University of Minnesota, Minneapolis, Minnesota, USA. ${ }^{4}$ Department of Genetics, Albert Einstein Medical Center, Philadelphia, Pennsylvania, USA. ${ }^{5}$ Medical Genetics Branch, National Human Genome Research Institute, National Institutes of Health, Bethesda, Maryland, USA. ${ }^{6}$ Genetics of Development and Disease Research Branch, National Institute of Diabetes and Digestive and Kidney Diseases, National Institutes of Health, Bethesda, Maryland, USA. ${ }^{7}$ Genetics Division, Children's National Medical Center, Washington, DC, USA. ${ }^{8}$ Weatherall Institute of Molecular Medicine, John Radcliffe Hospital, Oxford OX3 9DS, UK. ${ }^{9}$ Department of Medical Genetics, University Medical Centre Utrecht, Postbus 85090, 3508 AB Utrecht, the Netherlands. ${ }^{10}$ Department of Oral Pathology and Genetics, School of Dentistry, University of Minnesota, Minneapolis, Minnesota, USA. ${ }^{11}$ Genome Technology Branch, National Human Genome Research Institute, National Institutes of Health, Bethesda, Maryland, USA. ${ }^{12}$ Centre for Molecular Medicine, Stopford Building, Oxford Road, Manchester, UK. ${ }^{13}$ Manchester Royal Eye Hospital, Central Manchester and Manchester Children's University Hospitals NHS Trust, Oxford Road, Manchester, UK. Correspondence should be addressed to G.C.M.B. (gblack@man.ac.uk). 
a

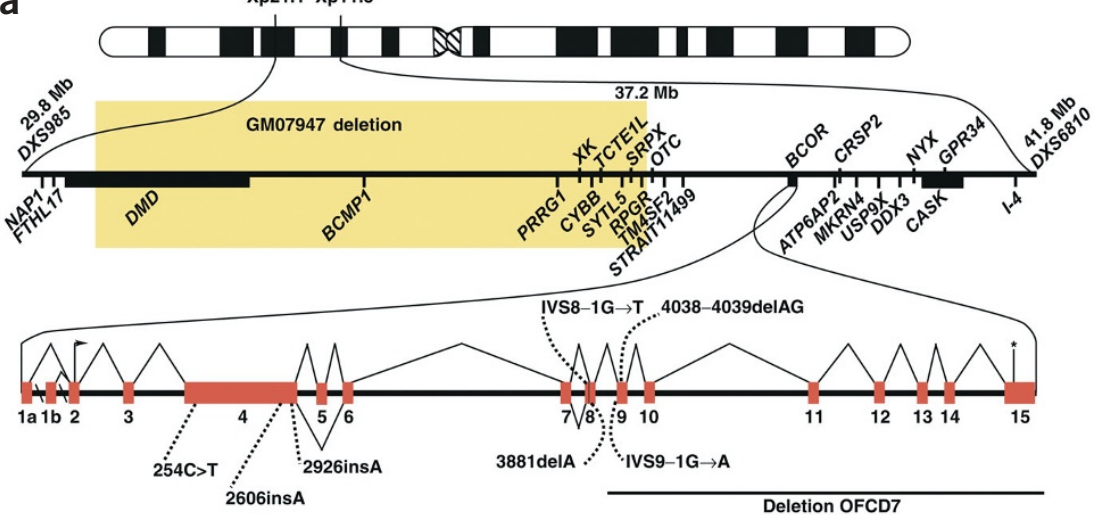

Mutated
Figure 1 Mapping of the MAA2 locus and mutations in the $B C O R$ gene. (a) Genetic mapping of the MAA2 locus identified a region flanked by DXS985 and DXS6810 (ref. 3). Deletion mapping excluded the region extending proximally to between RPGR and OTC. Sequencing showed a nt $254 \mathrm{C} \rightarrow$ T substitution in exon 4 of $B C O R$. Sequencing of females with OFCD from six families showed different termination, frameshift and splice site mutations. Individual OFCD7 had a deletion that included exons 9-15 of BCOR. (a-d) Examples of $B C O R$ point mutation electropherograms include exon $4 \mathrm{nt} 254 \mathrm{C} \rightarrow$ T substitution from an affected male with Lenz microphthalmia (b; see ref. 3 for pedigree); individual OFCD1, with an intron 8 splice-acceptor mutation (c); and individual OFCD2, with an intron 9 spliceacceptor mutation (d). Sequences of affected individual (left) and control (right) are shown with mutated bases arrowed. (e) Sequence alignment of the portion of the predicted human BCOR sequence that includes the conserved residue Pro85. This was aligned with that of four other sequences from GenBank and the D. rerio sequence cloned in this work.
As a single missense mutation did not satisfy our threshold for proof, we searched for an allelic form of syndromic microphthalmia. $\mathrm{OFCD}^{6-10}$ is a distinct form of syndromic microphthalmia with congenital cataracts, narrow face, broad nasal tip with separated cartilage, cleft palate, and cardiac and dental anomalies (canine radiculomegaly, root dilacerations, oligodontia and retained deciduous teeth; Fig. 2). OFCD is presumed to be inherited in an Xlinked dominant pattern with male lethality, and the phenotypic overlap led us to propose that it might be allelic with MAA2. We sequenced $B C O R$ in ten females from seven families with OFCD and found different mutations in all families (nonsense, frameshift, splicing and large deletion; Table 1). In two sporadic cases for which DNA from parents was available, the mutations
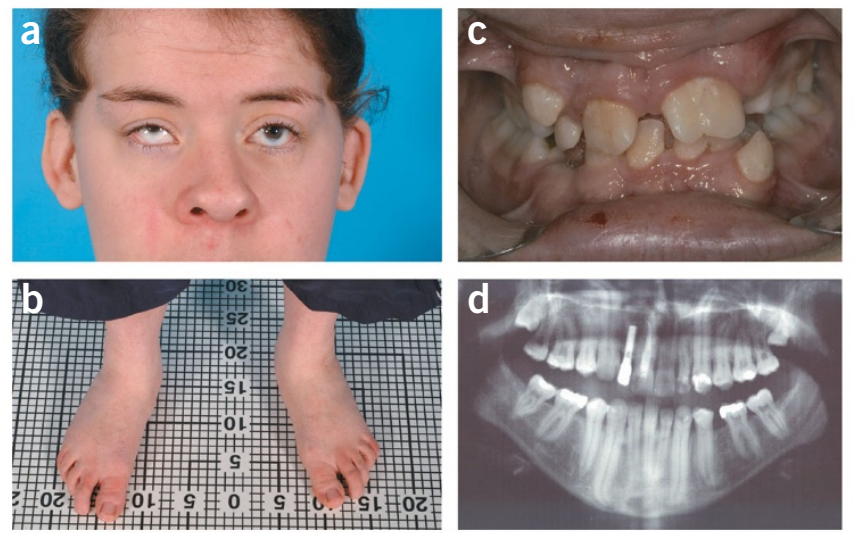

Figure 2 Clinical features of OFCD. (a-d) Features include mild microphthalmia and dysmorphic appearance with simple ears, elongated face and broad nasal tip (a), hammer-toe deformity (b), dental abnormalities with bilateral fused permanent upper incisors (c) and radiculomegaly of the lateral incisors shown on orthopantomogram (d). were de novo (paternity confirmed). In families OFCD5 and OFCD6, females in three and two generations, respectively, were affected and the mutations co-segregated.

The presence of truncating and frameshift mutations in seven families with OFCD and the identification of a missense mutation substituting a conserved proline that is absent in controls in a linked family with Lenz microphthalmia strongly supported the conclusion that mutations in BCOR cause both OFCD and one form of Lenz microphthalmia (associated with MAA2). In Lenz microphthalmia, a presumed hypomorphic mutation has severe phenotypic consequences in males. In OFCD, deletional, truncating and frameshift mutations are male-lethal and result in the 'milder' OFCD phenotype in females. Microphthalmia is less severe in OFCD than in Lenz syndrome, and in many females with OFCD the only ocular finding is cataract. In contrast, males in the family with Lenz syndrome have microphthalmia or anophthalmia, typically with microcephaly and structural brain anomalies. Females with OFCD are normal intellectually, but the aborted female fetus from family OFCD5 (OFCD5 individual 3, Table 1) had posterior fossa abnormalities. X-inactivation studies were informative in six individuals with OFCD and showed skewing (90-100\%) in all cases. Of these, both familial cases demonstrated preferential inactivation of the maternal chromosome (data not shown). This indicates that the mutations in $B C O R$ underlying OFCD may cause a selective disadvantage that in leukocytes results in skewing and in other tissues (BCOR is widely expressed) leads to variable phenotypic effects in a mosaic fashion, offering an explanation for the observation that OFCD in affected females is milder than Lenz syndrome in affected males. We predict that $B C O R$ forms a centromeric boundary for $\mathrm{Xp}$-contiguous gene syndromes, as a male with a deletion encompassing $B C O R$ should not be viable.

To further examine the pleiotropic effects of $B C O R$ mutations, we cloned the zebrafish $B C O R$ ortholog. We identified a 5,334nt open reading frame that corresponds to human $B C O R$ exons $4-15$. Mouse and human BCOR have $89 \%$ amino acid similarity, 
Table 1 Clinical details of individuals with Lenz microphthalmia and OFCD described in this study

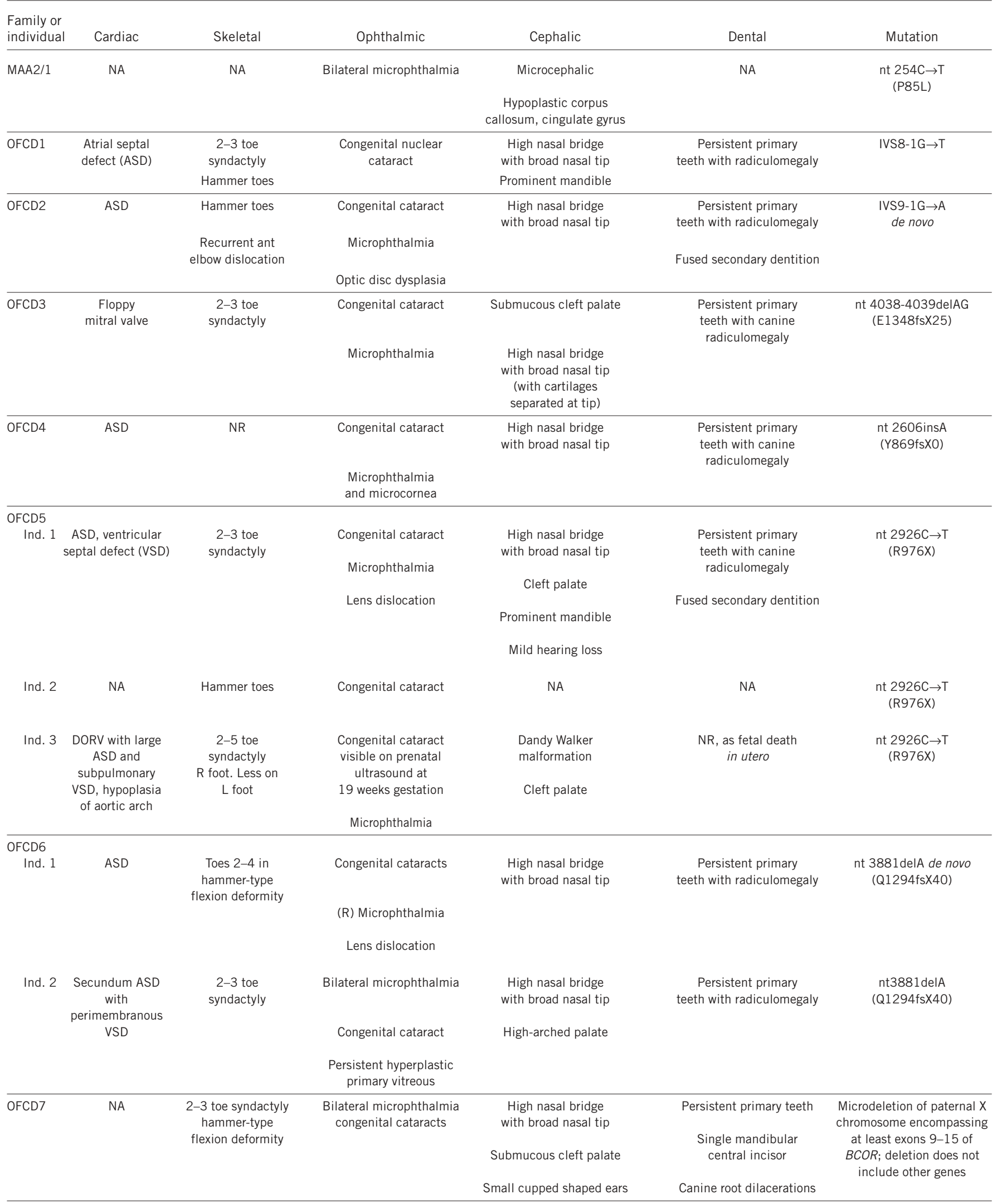

Several families have been previously described: Lenz microphthalmia ${ }^{3}$, OFCD5 (cases 1 and 2, ref. 7), OFCD6 (ref. 6). Family 5, case 3 , a female fetus, died in utero at 24 weeks gestation. All mutations numbered from beginning of reading frame in NM_017745. Ind., individual; NA, not affected; NR, not reported. 

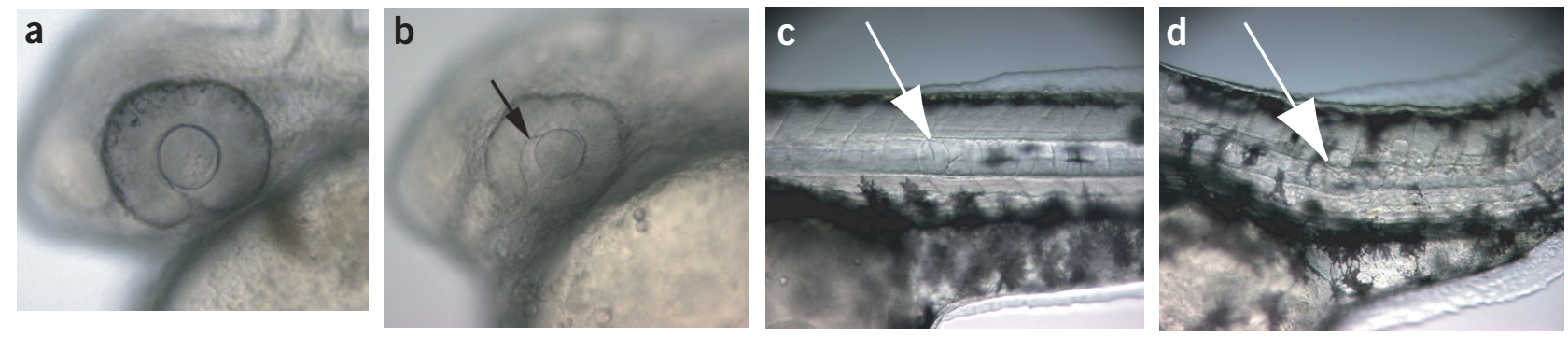

Figure 3 Morpholino knock-down experiments showed that loss of BCOR in the zebrafish recapitulates numerous human anomalies of Lenz microphthalmia and OFCD syndromes. (a) Normal eye development in fish embryos treated with control morpholinos. (b) Abnormal eye development, including colobomatous defects (arrow), in fish embryos injected with antisense morpholino (MO10). (c) Normal development of the neural tube in control-injected fish. Arrow shows an intact and regular neural tube floor plate. (d) Abnormal neural tube development, with irregularity of the floor plate, growth of notochord cells through the floor plate and invasion into the spinal cord (arrow), in fish embryos treated with antisense morpholino.

and we found $44 \%$ and $49 \%$ similarity between the mouse and human sequences, respectively, and that of the zebrafish. The Pro 85 residue in human BCOR is also conserved in the predicted zebrafish protein. The zebrafish sequence is most similar to human BCOR isoform D.

We identified zebrafish exon-intron boundaries from the trace database and found that the boundaries of human introns 3-15 are conserved in the zebrafish. We used antisense splice-blocking morpholino oligonucleotides ${ }^{11}$ to target the splice donor junctions of the zebrafish orthologs of human BCOR exons 9 and 10 (MO9, MO10), selected for their proximity to the human mutations. Examination of zebrafish embryos injected with 3-6 ms pulses of morpholino at 24 hours post fertilization (h.p.f.) showed severe central nervous system toxicity with necrosis, most evident with 6 ms of MO9. Morpholino MO10 caused more severe effects, and no embryos treated with $6 \mathrm{~ms}$ MO10 survived at 24 h.p.f. We therefore used $3 \mathrm{~ms}$ of MO10 for later experiments. A typical 3-ms MO10 experiment included 92 injected embryos, of which 84 survived $24 \mathrm{~h}$ and 79 of the 84 showed major morphologic defects. In contrast, the MO10MIS control included 43 injected embryos, of which 33 survived $24 \mathrm{~h}$ and only 2 had apparent morphologic defects $(P<0.0001$, Fisher's exact test). These MO10-treated zebrafish had colobomatous eye defects (Fig. 3a,b) and skeletal defects including disarrayed somites, shortening and kyphotic tails (Fig. 3c,d). They a

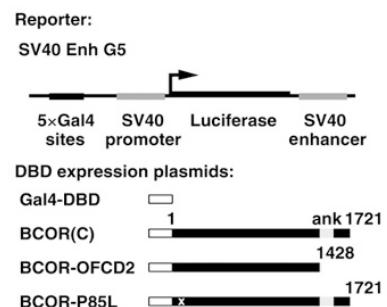

b

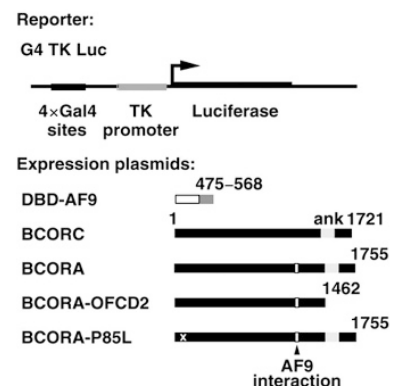

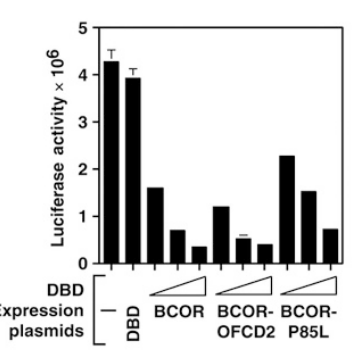

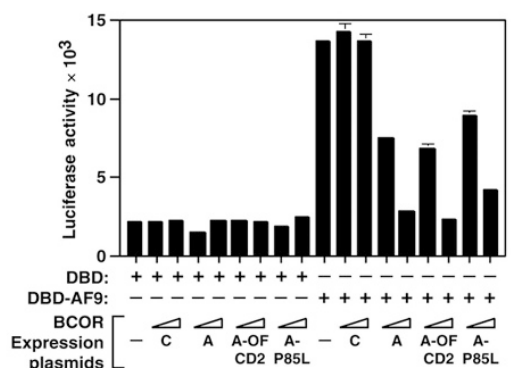

C

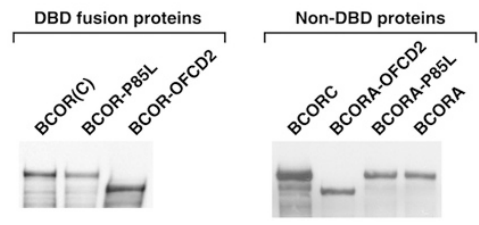

d

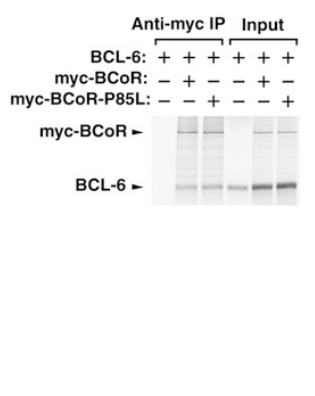

Figure 4 Biochemical studies of BCOR function. (a,b) Luciferase and $\beta$-galactosidase reporter assays. Left, reporter construct and proteins; right, data averaged over three experiments, with s.d. Ank, ankyrin repeats. (a) Assays were carried out on extracts from HeLa cells transiently transfected with 50 ng GAL4-responsive reporter (SV40 Enh G5), 0, 5, 15 or $45 \mathrm{ng}$ of GAL4 DBD fusion constructs, and $25 \mathrm{ng}$ pCMVLacZ plasmid. Luciferase activity was normalized to $\beta$-galactosidase levels. (b) BCOR isoform A, but not C, contains an AF9 interaction region, providing specificity for both interaction and repression. Assays were done using $5 \mathrm{ng}$ GAL4-responsive reporter (G4 TK Luc), $5 \mathrm{ng}$ of DBD-AF9(475-568) or DBD, and 0, 2 or $15 \mathrm{ng}$ of the indicated BCOR constructs and $25 \mathrm{ng} \mathrm{pCMVLacZ} \mathrm{plasmid.} \mathrm{(c)} \mathrm{Western} \mathrm{analysis} \mathrm{of} \mathrm{expression} \mathrm{plasmids} \mathrm{used} \mathrm{in} \mathbf{a}$ and $\mathbf{b}$ showing similar amounts of expression of wildtype and mutant BCOR. (d) In vitro coimmunoprecipitations showing normal interaction of P85L mutant BCOR with BCL-6. 
also had developmental defects of the central nervous system, including disruption and irregularities of the cerebellum, optic tectum and boundaries of the fourth ventricle, and growth of notochord cells through the floor plate and invasion into the spinal cord, as seen with MO9 at 72 h.p.f.. Control morpholinos, MO9MIS and MO10MIS, with 5-base-pair mismatches produced very few abnormal developmental effects. These data indicate that loss of function of zebrafish BCOR causes pleiotropic defects reminiscent of Lenz microphthalmia and OFCD syndromes.

We also investigated the functional consequences of the OFCD and Lenz mutations. BCOR is a transcriptional corepressor identified through yeast two-hybrid screens ${ }^{4}$ for interaction with the POZ domain of BCL-6, a transcription factor identified in translocations in non-Hodgkin's lymphoma ${ }^{12-15}$. BCL-6-deficient mice develop inflammatory disease and have defects in T cell-dependent antibody responses ${ }^{16-18}$. In addition, specific BCOR isoforms interact with the MLL (mixed lineage leukemia) fusion partner AF9 and function as a corepressor for the AF9 (Mllt3) activation domain ${ }^{19}$. MLL is a developmental regulator homologous to the $D$. melanogaster gene trx (trithorax). We tested corepressor function (i) by tethering BCOR to a promoter and measuring its ability to repress the SV40 enhancer (Fig. 4a) and (ii) by measuring BCOR's ability to repress a tethered AF9 activation domain (Fig. 4b). We compared wild-type BCOR to a partially deleted BCOR that mimics the predicted truncation product of individual OFCD2 expressed at similar levels (Fig. 4c). In both assays the BCOR protein from OFCD2 showed repressor effects equivalent to those of wild-type BCOR (Fig. 4a,b). All the mutations identified in individuals with OFCD predict premature stop codons and have similar phenotypic consequences. It is likely that nonsense-mediated mRNA decay prevents the truncated BCOR proteins from being expressed in vivo, but this cannot be tested because of the severe skewing of X inactivation in individuals with OFCD. In the case of the P85L mutant, nonsense-mediated mRNA decay is unlikely. BCOR P85L interacted normally with BCL-6 (Fig. 4d) and showed normal or near-normal repressive activity in two assays (Fig. $\mathbf{4 a}, \mathbf{b})$. The slightly lower activity of $\mathrm{P} 85 \mathrm{~L}$ in the tethered BCOR assay was probably due to lower protein abundance (Fig. $4 \mathbf{c}$ ).

These results, together with the lack of an ocular phenotype ${ }^{16-18}$ (or other manifestations of Lenz microphthalmia or OFCD syndromes) in BCL-6-deficient mice, indicate that transcriptional regulators other than BCL-6 are likely to participate with BCOR in the development of a range of nonimmunologic systems. This is supported by the ubiquitous expression pattern of BCOR in both fetal and adult tissues ${ }^{4}$. That individuals with Lenz microphthalmia have no immunoregulatory deficits also indicates that mutations in BCOR have developmental consequences that involve non-BCL-6 pathways.

We propose that additional transcription factors interact with BCOR in the developing eye. Complex regulatory networks of transcriptional activators and repressors have now been defined in eye development ${ }^{20}$ and offer several attractive candidates. Cataract is the most constant ocular phenotype of OFCD and several lens-specific gene products $(\alpha-$ and $\beta$-crystallins, MIP, PAX6, PITX3 and MAF) are required for both normal ocular patterning and lens formation ${ }^{21}$. We suggest that PAX6, MAF and SOX1-3 may act as coregulators with, or counter-regulators of, BCOR. MAF is crucial for regulation of lens crystallin expression and is activated by CREB-binding protein (which is haploinsufficient in Rubinstein-Taybi syndrome, a pleiotropic developmental disorder that can be associated with both cataract and microphthalmia ${ }^{22}$ ) and p300 in the presence of histone acetylase activity ${ }^{23}$. Notably, BCOR interacts with class I and II histone deacetylases ${ }^{4}$ and p300 binds and acetylates
BCL- 6 to inhibit recruitment of histone deacetylases and the ability of BCL- 6 to repress transcription ${ }^{24}$. Taken together, these data suggest that BCOR, which is crucial for ocular and nonocular patterning, is involved in transcriptional repression, possibly mediated through the modulation of histone acetylation and subsequent chromatin remodeling.

\section{METHODS}

Human and animal subjects. This study was done as part of a protocol reviewed and approved by the National Human Genome Research Institutional Review Board and the Research Ethics committee at Manchester University, and informed consent was obtained from all participants. The zebrafish studies were carried out under a protocol reviewed and approved by the NHGRI animal care and use committee.

Deletion mapping. After extracting genomic DNA from Coriell GM07947 (Coriell Institute) with QIAmp DNA Blood Mini Kit (Qiagen), we identified intragenic microsatellite repeats (STRs) in DMD, PRRG1, XK, TCTE1L, SRPX, OTC, TM4SF2 and STRAIT11499. STS analysis was done using standard techniques. The Xp interstitial deletion of individual OFCD7 was defined using short tandem repeat polymorphisms located in BACs AC0921928 and AC091809 (primer sequences are available upon request).

Mutation analysis. We amplified the coding exons of $B C O R$, including the flanking intronic sequence, from genomic DNA that was isolated using affinity chromatography (Qiagen). We carried out PCR using Herculase DNA polymerase and Perfect Match PCR enhancer (Stratagene) with a PTC-100 Programmable Thermal Controller (MJ Research). An initial denaturation step of $3 \mathrm{~min}$ at $98^{\circ} \mathrm{C}$ was followed by 40 cycles of amplification $\left(1 \mathrm{~min}\right.$ at $98^{\circ} \mathrm{C} ; 1$ min at $50{ }^{\circ} \mathrm{C} ; 1 \mathrm{~min} / \mathrm{kb}$ at $72{ }^{\circ} \mathrm{C}$ ) and a final extension at $72{ }^{\circ} \mathrm{C}$ for $10 \mathrm{~min}$. We sequenced PCR products with the dGTP BigDye Terminator ready reaction kit (PE Applied Biosystems) on an ABI 377 Prism automated sequencer (PE Applied Biosystems; 5\% LongRanger acrylamide gel, BMA) and analyzed the data using ABI Prism Sequencing Analysis 3.0 and Sequencher 4.1 software (GeneCodes). We compared the sequence data with published BCOR sequence (GenBank reference numbers AF317391 and AL591802) and controls. Although $B C O R$ has alternative splicing and previously unrecognized splice forms were identified in the course of this work, seven of eight mutations we identified are in exons that are invariant (one mutation associated with OFCD, IVS8- $1 \mathrm{G} \rightarrow \mathrm{T}$, is at a variable splice acceptor).

We analyzed controls for the nt $254 \mathrm{C} \rightarrow \mathrm{T}$ mutation by PCR followed by restriction digestion of the product; the mutation removes a SmaI restriction site. Controls included a panel of 100 samples derived from African American individuals (83 female and 17 male), a panel of 92 samples from African American individuals of unknown sex (estimated to be $50 \%$ female) and a panel of 92 samples derived from individuals of mixed ethnicity, 46 each males and females.

Zebrafish RT-PCR and $3^{\prime}$ and $5^{\prime}$ RACE. We cloned the zebrafish $B C O R$ ortholog by rapid amplification of cDNA ends (RACE) starting from a single expressed sequence tag (EST), AI354165. We extracted RNA from adult zebrafish tails with RNAeasy (Qiagen) according to the manufacturer's instruction. We carried out first-strand cDNA synthesis with the SMART RACE cDNA amplification kit (BD Biosciences Clontech) and $3^{\prime}$ and $5^{\prime}$ RACE PCR with the Advantage-GC 2 PCR kit (BD Biosciences Clontech) using custom nested primers (Invitrogen) designed from EST AI354165. PCR products were gel purified with a Qiagen gel purification kit and sequenced with an ABI 377 Prism automated sequencer. Sequence data were analyzed with ABI Sequencing analysis 3.0 and Sequencher 4.1 software. No similar paralogs in zebrafish were detected, suggesting that this zebrafish gene has not been duplicated.

Morpholinos. We designed antisense splice-blocking morpholino oligonucleotides (which we obtained from Gene Tools) based on $5^{\prime}$ RACE sequence to target zebrafish BCOR orthologs of human exons 9 and 10 by annealing to the splice donors of each exon with extension into the adjoining intronic sequence. Sequences of the morpholinos are available on request. We resuspended the morpholinos with purified water at $300 \mathrm{nM}$ concentration, diluted them to $75 \mathrm{nM}$ for injections, and injected $\sim 0.5 \mathrm{ng}$ into the yolk sac of the embryo as described ${ }^{11}$. 
X-inactivation studies. We analyzed X-inactivation patterns by methylation PCR with bisulfite modification of genomic DNA of the trinucleotide repeat in exon 1 of the human androgen receptor ${ }^{25,26}$. We used FAM-labeled primers to label the products and carried out fragment analysis on an ABI 377 Prism with Genescan 3.1 software. Peak area correction was done as described ${ }^{25}$.

Biochemical studies. We generated the P85L mutation in the $\mathrm{C}$ isoform of EFmycBCOR, pT7-myc BCOR and pCMV-DBD-BCOR ${ }^{4}$ using the Promega gene editor kit. The genomic IVS11-1G $\rightarrow$ A mutation of individual OFCD2 was assumed to splice from exon 11 to exon 13, generating an in-frame stop codon 6 amino acids downstream of the splice junction. We generated a cDNA fragment representing this new $\mathrm{C}$ terminus by PCR. All primer sequences are available on request. We digested the product with BsmI and NotI and cloned it into an intermediate vector to pick up an XbaI site beyond the NotI site. Next, we cloned the $B s m \mathrm{I}$ and $\mathrm{XbaI}$ fragment from this vector into BsmI- (partial) and $\mathrm{XbaI}$-cut CMV-DBD-BCOR, and substituted a PmlI-XbaI fragment from this into the $E F m y c B C O R$ vector. We generated the $B C O R$ A isoform region by PCR from cDNA inserted into the $\mathrm{C}$ isoform EFBCOR. We transferred a BsmI fragment from this vector into all other A isoform $B C O R$ vectors. The PCR-generated sequences were confirmed by sequencing. DBD-BCOR repression assays and cotranslation coimmunoprecipitation assays were as described ${ }^{4}$. The assay of BCOR repression of DBD-AF9 was similar to one used previously ${ }^{19}$ except that we used human and not mouse proteins and the reporter contained the thymidine kinase (TK) promoter and no SV40 enhancer. We derived the C-terminal 94 amino acids of human $A F 9$ by PCR from a cDNA generously provided by $\mathrm{J}$. Kersey (University of Minnesota) and cloned into CMV-DBD. Expression promoter concentrations were kept constant in a given experiment by the inclusion of empty expression vectors, either EFmyc or CMV-DBD. We carried out transfection of 293 cells using $\mathrm{CaPO}_{4}$ in 6-cm dishes. For western analysis, we transfected 293 cells with $6 \mu \mathrm{g}$ of the indicated EFmycBCOR or GAL4 DBD fusion constructs and $400 \mathrm{ng}$ of pEFLacZ plasmid. Whole cell extracts with equivalent $\beta$-galactosidase activity were blotted with an anti-BCOR antibody (V.J.B., unpublished results). For in vitro coimmunoprecipitations, we produced $\left[{ }^{35} \mathrm{~S}\right]$ methionine-labeled, untagged BCL-6 and myc-tagged BCOR proteins in vitro (Promega Quick Coupled System), immunoprecipitated them with antimyc antibody ${ }^{4}$, recovered them and analyzed them by $10 \%$ SDS-PAGE.

Accession numbers. BCL-6 interacting corepressor is NM_017745; this is the ortholog of Mus musculus BCOR isoform D, AY161173. Human BCOR is AY316593. The Takifugu rubripes sequence was predicted from CAAB01000660, Rattus norvegicus BCOR is XP_343768 and the Gallus gallus BCOR protein sequence was predicted from an EST contig comprised of BU451897, BU402004 and BU234234. The D. rerio BCOR cDNA cloned in this work is AY316593.

\section{ACKNOWLEDGMENTS}

The authors thank the families with Lenz microphthalmia and OFCD who generously volunteered to participate in this research project; T. Wolfsberg for bioinformatics assistance with identifying EST AI354165; R. Kittles for control DNA specimens; L. Samuels for assistance with contacting one affected individual; E. Tsilou and B. Rubin for ophthalmologic assessment of an individual with OFCD; J. Johnston for analyzing a set of controls for the nt $254 \mathrm{C} \rightarrow \mathrm{T}$ mutation; and D. Zarkower for critical reading of the manuscript. This work was supported by funding from the Division of Intramural Research of the National Human Genome Research Institute, National Institutes of Health (L.G.B.) and an NIH extramural grant (V.J.B.). G.C.M.B., a Senior Research Fellow in Clinical Science, and R.P. are funded by the Wellcome Trust.

\section{COMPETING INTERESTS STATEMENT}

The authors declare that they have no competing financial interests.
Received 26 August 2003; accepted 30 January 2004

Published online at http://www.nature.com/naturegenetics/

1. Graham, C.A., Redmond, R.M. \& Nevin, N.C. X-linked clinical anophthalmos. Localization of the gene to Xq27-Xq28. Ophthalmic Paediatr. Genet. 12, 43-48 (1991).

2. Forrester, S., Kovach, M.J., Reynolds, N.M., Urban, R. \& Kimonis, V. Manifestations in four males with and an obligate carrier of the Lenz microphthalmia syndrome. Am. J. Med. Genet. 98, 92-100 (2001).

3. Ng, D., Hadley, D.W., Tifft, C.J. \& Biesecker, L.G. Genetic heterogeneity of syndromic X-linked recessive microphthalmia-anophthalmia: is Lenz microphthalmia a single disorder? Am. J. Med. Genet. 110, 308-314 (2002).

4. Huynh, K.D., Fischle, W., Verdin, E. \& Bardwell, V.J. BCoR, a novel corepressor involved in BCL-6 repression. Genes Dev. 14, 1810-1823 (2000).

5. Brown, J. et al. Analysis of three deletion breakpoints in Xp21.1 and the further localization of RP3. Genomics 37, 200-210 (1996).

6. Wilkie, A.O., Taylor, D., Scambler, P.J. \& Baraitser, M. Congenital cataract, microphthalmia and septal heart defect in two generations: a new syndrome? Clin. Dysmorphol. 2, 114-119 (1993).

7. Aalfs, C.M. et al. Cataracts, radiculomegaly, septal heart defects and hearing loss in two unrelated adult females with normal intelligence and similar facial appearance: confirmation of a syndrome? Clin. Dysmorphol. 5, 93-103 (1996).

8. Schulze, B.R., Horn, D., Kobelt, A., Tariverdian, G. \& Stellzig, A. Rare dental abnormalities seen in oculo-facio-cardio-dental (OFCD) syndrome: three new cases and review of nine patients. Am. J. Med. Genet. 82, 429-435 (1999).

9. Gorlin, R.J., Marashi, A.H. \& Obwegeser, H.L. Oculo-facio-cardio-dental (OFCD) syndrome. Am. J Med Genet 63, 290-292 (1996).

10. Hedera, P. \& Gorski, J.L. Oculo-facio-cardio-dental syndrome: skewed X chromosome inactivation in mother and daughter suggest X-linked dominant inheritance. Am. J. Med. Genet. 123A, 261-266 (2003).

11. Nasevicius, A. \& Ekker, S.C. Effective targeted gene 'knockdown' in zebrafish. Nat. Genet. 26, 216-220 (2000).

12. Baron, B.W. et al. Identification of the gene associated with the recurring chromosomal translocations $\mathrm{t}(3 ; 14)(\mathrm{q} 27 ; \mathrm{q} 32)$ and $\mathrm{t}(3 ; 22)(\mathrm{q} 27 ; \mathrm{q} 11)$ in B-cell lymphomas. Proc. Natl. Acad. Sci. USA 90, 5262-5266 (1993).

13. Kerckaert, J.P. et al. LAZ3, a novel zinc-finger encoding gene, is disrupted by recurring chromosome 3q27 translocations in human lymphomas. Nat. Genet. 5, 66-70 (1993).

14. Miki, T. et al. Molecular cloning of the breakpoint for $3 q 27$ translocation in B-cell lymphomas and leukemias. Blood 83, 217-222 (1994).

15. Ye, B.H., Rao, P.H., Chaganti, R.S. \& Dalla-Favera, R. Cloning of bcl-6, the locus involved in chromosome translocations affecting band 3q27 in B-cell lymphoma. Cancer Res. 53, 2732-2735 (1993).

16. Dent, A.L., Shaffer, A.L., Yu, X., Allman, D. \& Staudt, L.M. Control of inflammation, cytokine expression, and germinal center formation by BCL-6. Science 276, 589-592 (1997).

17. Ye, B.H. et al. The BCL-6 proto-oncogene controls germinal-centre formation and Th2-type inflammation. Nat. Genet. 16, 161-170 (1997).

18. Fukuda, T. et al. Disruption of the Bcl6 gene results in an impaired germinal center formation. J. Exp. Med. 186, 439-448 (1997).

19. Srinivasan, R.S., de Erkenez, A.C. \& Hemenway, C.S. The mixed lineage leukemia fusion partner AF9 binds specific isoforms of the BCL-6 corepressor. Oncogene 22 3395-3406 (2003).

20. Epstein, J.A. \& Neel, B.G. Signal transduction: an eye on organ development. Nature 426, 238-239 (2003).

21. Graw, J. The genetic and molecular basis of congenital eye defects. Nat. Rev. Genet. 4, 876-888 (2003)

22. van Genderen, M.M., Kinds, G.F., Riemslag, F.C. \& Hennekam, R.C. Ocular features in Rubinstein-Taybi syndrome: investigation of 24 patients and review of the literature. Br. J. Ophthalmol. 84, 1177-1184 (2000).

23. Chen, Q., Dowhan, D.H., Liang, D., Moore, D.D. \& Overbeek, P.A. CREB-binding protein/p300 co-activation of crystallin gene expression. J. Biol. Chem. 277, 24081-24089 (2002).

24. Bereshchenko, O.R., Gu, W. \& Dalla-Favera, R. Acetylation inactivates the transcriptional repressor BCL6. Nat. Genet. 32, 606-613 (2002).

25. Kubota, T. et al. A new assay for the analysis of X-chromosome inactivation based on methylation-specific PCR. Hum. Genet. 104, 49-55 (1999).

26. Allen, R.C., Zoghbi, H.Y., Moseley, A.B., Rosenblatt, H.M. \& Belmont, J.W. Methylation of Hpall and Hhal sites near the polymorphic CAG repeat in the human androgen-receptor gene correlates with $\mathrm{X}$ chromosome inactivation. Am. J. Hum. Genet. 51, 1229-1239 (1992). 